\title{
Evidence for a superantigen mediated process in Kawasaki disease
}

\author{
Nigel Curtis, Richard Zheng, Jonathan R Lamb, Michael Levin
}

\begin{abstract}
The clinical, pathological, and immunological similarities between Kawasaki disease and the staphylococcal and streptococcal toxic shock syndromes suggest that a superantigen toxin may be involved in the pathogenesis of the disease. The $V \beta$ repertoire of peripheral blood mononuclear cells from 21 children with Kawasaki disease, 28 children with other illnesses, and 22 healthy controls were examined using monoclonal antibodies to $V \beta 2,5,8,12$, and 19. The mean percentage of $\mathrm{V} \beta 2$ expressing $T$ cells in the patients with Kawasaki disease was increased when compared with healthy controls or children with other illnesses. The mean percentages of $V \beta 5,8,12$, and 19 expressing $T$ cells were also increased in the patients with Kawasaki disease compared with healthy controls, but were not increased when compared with children with other illnesses. The selective use of $\mathrm{V} \beta 2$ supports the hypothesis that a superantigen is involved in the pathogenesis of Kawasaki disease.
\end{abstract}

(Arch Dis Child 1995; 72: 308-311)

Keywords: Kawasaki disease, superantigen, $\mathrm{T}$ cell receptor.

In the 25 years since the first description of Kawasaki disease in Japan, ${ }^{1}$ the disorder has become recognised world wide as an important disease of infancy and early childhood. In the UK the minimum estimate of the incidence of the disease is $3 \cdot 4 / 100000$ children under 5 years, ${ }^{2}$ which is similar to that in the $\mathrm{US}^{3}$ but less than $10 \%$ of that in Japan $(172 / 100000$ children under 5). ${ }^{4}$ Although in most cases the disease is a self limiting febrile exanthematous illness, coronary artery abnormalities due to an inflammatory arteritis develop in $15-30 \%$ of patients. ${ }^{56}$ Myocardial infarction or aneurysm rupture account for death in $1-3 \%$ of cases. ${ }^{2} 7$

The epidemiological features of the disorder clearly suggest an infectious aetiology: the disorder affects a defined age group of children with a peak at 1 year ${ }^{4}$; epidemics and clustering of cases are commonly observed ${ }^{9}$; and wave-like spread from one part of the country to another has been reported. ${ }^{10}$ Secondary cases are rare, but do occur in family members with an 8-9\% incidence in siblings under 2 years. ${ }^{11}$ These epidemiological features are most consistent with an infectious agent that is widely distributed in the population, to which most older children and adults are immune, and which causes disease expression in only a small percentage of susceptible individuals.

A variety of different organisms have been suggested as the cause, including mite associated bacteria, ${ }^{12}$ rickettsia, ${ }^{13}$ the anaerobic organism Propionibacterium acnes, ${ }^{14}$ several viruses including parvovirus $\mathrm{B} 19,{ }^{15}$ and retroviruses. ${ }^{1617}$ Promising initial reports implicating each of these organisms have not been confirmed on subsequent studies.

There are striking similarities between Kawasaki disease, and the staphylococcal and streptococcal toxic shock syndromes ${ }^{18-20}$ and scarlet fever. Each of these diseases is associated with high fever, an erythematous rash with desquamation in the convalescent phase, mucous membrane and conjunctival inflammation, and an intense acute phase response. The staphylococcal and streptococcal toxin disorders are now known to be caused by a family of protein toxins which have considerable
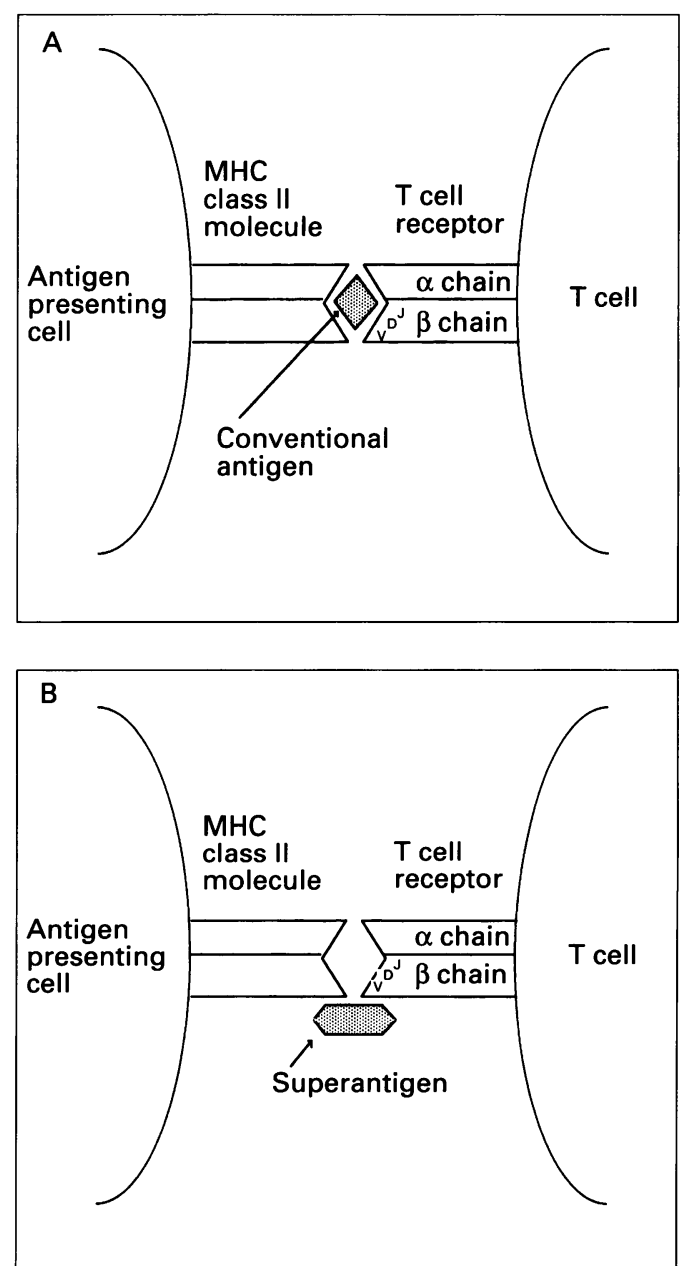

Figure 1 (A) $T$ cell stimulation by a conventional antigen. (B) $T$ cell stimulation by a superantigen. 
homology and a common mode of action. The ability of these proteins to cause intense $T$ cell activation has led to their classification as superantigens. ${ }^{21} 22$

A conventional antigen is first processed by an antigen presenting cell (APC) and then presented as a peptide on the APC surface in association with a major histocompatibility complex (MHC) class II molecule (fig 1A). This complex is recognised by the few (about 1 in $10^{6}$ ) $\mathrm{T}$ cells which bear specific receptors for this antigen. This highly specific interaction involves the variable $(V)$, joining $(J)$, and diversity (D) portions of both the $\alpha$ and $\beta$ chains of the $T$ cell receptor (TCR).

In contrast to conventional antigens, superantigens are able to stimulate a vast number of $\mathrm{T}$ cells by directly binding class II molecules on APCs and the TCR (fig 1B). This interaction is restricted only by the specificity of the variable region of the $\beta$ chain $(V \beta)$ of the TCR. Any given superantigen stimulates a specific set of V $\beta$ families; for example, staphylococcal toxic shock syndrome is caused by toxic shock syndrome toxin which stimulates all $\mathrm{T}$ cells bearing $V \beta 2$. As there are only a limited number of $V \beta$ families, a superantigen may stimulate a large proportion of all $\mathrm{T}$ cells. This intense $\mathrm{T}$ cell proliferation and consequent immune activation, including cytokine release, may be central to the pathogenesis of toxic shock syndrome.

In view of the clinical and pathological similarities between Kawasaki disease and the disorders caused by the superantigen toxins, we postulated, ${ }^{23}$ as have others, ${ }^{24} 25$ that Kawasaki disease might be caused by a superantigen toxin produced by micro-organisms present in the respiratory or gastrointestinal tract of affected patients. In order to investigate this hypothesis we studied the pattern of $V \beta$ receptor usage in patients with Kawasaki disease and controls and here present evidence which supports the possibility of a superantigen mediated process.

\section{Patients and methods \\ PATIENTS}

We studied 21 children fulfilling the diagnostic criteria for Kawasaki disease ${ }^{26}$ who presented to the infectious diseases unit at St Mary's Hospital, or to collaborating centres in England. Patients who had less than four of the diagnostic criteria for Kawasaki disease were included in the study if they had coronary artery dilatation or aneurysms. Patients were excluded if they had evidence of shock or if group A streptococcus was isolated or detected serologically, unless they had evidence of coronary artery aneurysms. Venous blood was collected from the patients into one tenth volume trisodium citrate as soon as possible after admission. Blood samples were also obtained where possible during the convalescent phase of the illness.

Twenty eight other children were studied, who were admitted to St Mary's Hospital with a range of childhood illnesses, including both bacterial and viral infections. Samples were also obtained from 22 healthy adult laboratory or clinical personnel.

The study was approved by the St Mary's Hospital ethics committee.

\section{ANALYSIS OF V $\beta$ RECEPTOR USAGE}

The proportion of peripheral blood mononuclear cells (PBMCs) expressing particular $T$ cell receptor $V \beta$ chain families was studied using available monoclonal antibodies against $\mathrm{V} \beta 2,5,8,12$, and 19. PBMCs were isolated by gradient centrifugation on Ficoll-Hypaque (Pharmacia), and the cells washed in phosphate buffered saline. The cells were blocked with $10 \%$ normal mouse serum (Sigma) for one hour at $4^{\circ} \mathrm{C}$, and incubated with fluoroscein isothiocyanate (FITC) conjugated monoclonal antibodies against V $2,8,19$ (Immunotech), V $\beta 5,12$ (T Cell Diagnostics), CD3 (Immunotech) or non-specific FITC conjugated IgG (Immunotech and $T$ Cell Diagnostics), at the manufacturer's recommended concentration for half an hour at $4^{\circ} \mathrm{C}$. The cells were then washed three times and the proportion of cells expressing each of the V $\beta$ families analysed by flow cytometry using an Epics-Profile II counter (Coulter Electronics). The results for each $V \beta$ family were expressed as a proportion of the total CD3 positive cells.

To validate the method for detecting changes in the proportion of cells expressing individual V $\beta$ families, PBMCs from five healthy adult donors were cultured at a density of $2 \times 10^{6} / \mathrm{ml}$ in RPMI medium (Gibco) and $10 \%$ fetal calf serum (Gibco) in the presence or absence of $10 \mu \mathrm{g} / \mathrm{ml}$ anti-CD3, $0.01 \mu \mathrm{g} / \mathrm{ml}$ purified toxic shock syndrome toxin (Sigma) or $0.01 \mu \mathrm{g} / \mathrm{ml}$ purified staphylococcal enterotoxin B (Sigma). The cells were cultured at $37^{\circ} \mathrm{C}$ and were stimulated with interleukin-2 (IL-2) (Lymphocult T; 10\% vol/vol, Biotest Folex) on day 3 and day 7 . The cells were harvested on day 8 and the proportion of each of the V $\beta$ families analysed as described above.

\section{STATISTICS}

The differences in the proportion of each $\mathrm{V} \beta$ family in the patients with Kawasaki disease and the controls were compared using the Mann-Whitney U test.

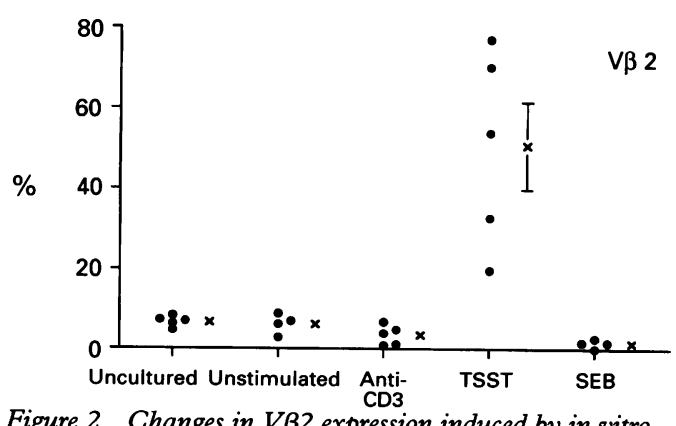

Figure 2 Changes in VB2 expression induced by in vitro stimulation with toxic shock syndrome toxin (TSST) or staphylococcal enterotoxin $B$ (SEB). The mean percentage of $V \beta 2$ expressing $P B M C$ s was significantly increased after stimulation with TSST when compared with uncultured $(p<0.01)$ or unstimulated PBMCs $(p<0.05)$, or those stimulated with anti-CD3 $(p<0.01)$ or $S E B(p<0.05)$. Each point represents an individual experiment. Crosses represent means with SEM indicated by bars. 


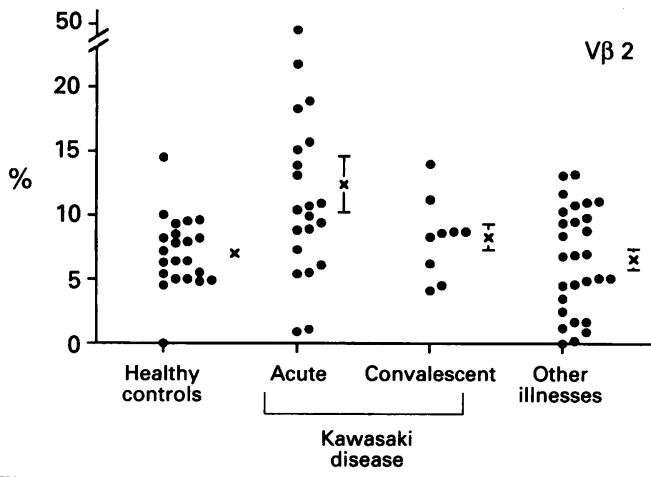

Figure $3 \quad V \beta 2$ expressing $T$ cells in patients with Kawasaki disease and controls. The mean percentage of $V \beta 2$ expressing $T$ cells was significantly increased in the Kawasaki disease patients when compared with healthy controls $(p<0.01)$ or those with other illnesses $(p<0.01)$ Each point represents an individual patient. Crosses represent means with SEM indicated by bars.

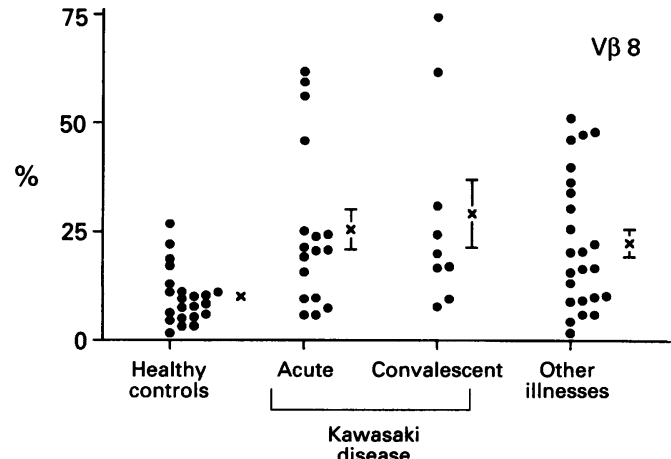

Figure $4 V \beta 8$ expressing $T$ cells in patients with Kawasaki disease and controls. The mean percentage of $V \beta 8$ expressing $T$ cells was significantly increased in the Kawasaki disease patients when compared with healthy controls $(p<0.005)$ but was not significantly different from convalescent Kawasaki disease patients $(p=0.73)$ or those with other illnesses $(p=0.67)$. Each point represents an individual patient. Crosses represent means with SEM indicated by bars.

\section{Results}

When normal PBMCs were stimulated with toxic shock syndrome toxin there was a significant rise in the proportion of V $\beta 2$ bearing $\mathrm{T}$ cells (Fig 2). The proportion of V 22 expressing $T$ cells in the peripheral blood of patients with acute Kawasaki disease (mean (SEM) (12.5 $(2 \cdot 2) \%)$ was significantly higher than that in children with other illnesses $(6.6 \quad(0.8) \%$, $\mathrm{p}<0.01)$ or healthy controls $(7.0(0.6 \%)$, $\mathrm{p}<0.01)$. Although the $\mathrm{V} \beta 2$ expression in patients with acute Kawasaki disease was higher than that in patients convalescing from the illness, this did not reach statistical significance $(8 \cdot 3(1 \cdot 0) \%, p=0 \cdot 13)$ (fig 3$)$.

The proportion of $\mathrm{V} \beta 8$ positive $T$ cells in the patients with Kawasaki disease was also

Mean (SEM) percentage of CD3 positive PBMCs expressing each of the VB families (V $\beta$ $2,5,8,12,19)$ studied; $n=$ number of patients from whom $P B M C$ s were obtained in sufficient numbers for analysis for each $V \beta$ family

\begin{tabular}{|c|c|c|c|c|}
\hline & \multirow[b]{2}{*}{ Healthy controls } & \multicolumn{2}{|l|}{ Kawasaki disease } & \multirow[b]{2}{*}{ Other illnesses } \\
\hline & & Acute & Convalescent & \\
\hline $\begin{array}{l}V \beta 2 \\
V \beta 5 \\
V \beta 8 \\
V \beta 12 \\
V \beta 19\end{array}$ & $\begin{array}{r}7 \cdot 0(0 \cdot 6)[n=22] \\
5 \cdot 0(0 \cdot 5)[n=21] \\
10 \cdot 0(1 \cdot 4)[n=22] \\
5 \cdot 8(1 \cdot 2)[n=20] \\
5 \cdot 1(0 \cdot 5)[n=22]\end{array}$ & $\begin{array}{r}12 \cdot 5(2 \cdot 2)[n=21] \\
18 \cdot 4(3 \cdot 2)[n=20] \\
25 \cdot 5(4 \cdot 6)[n=17] \\
19 \cdot 0(4 \cdot 0)[n=20] \\
8 \cdot 8(0 \cdot 9)[n=21]\end{array}$ & $\begin{array}{r}8 \cdot 3(1 \cdot 0)[n=9] \\
14 \cdot 6(2 \cdot 9)[n=8] \\
29 \cdot 2(7 \cdot 8)[n=9] \\
20 \cdot 1(3 \cdot 9)[n=9] \\
5 \cdot 5(0 \cdot 7)[n=9]\end{array}$ & $\begin{array}{r}6 \cdot 6(0 \cdot 8)[n=28] \\
15 \cdot 5(3 \cdot 9)[n=24] \\
22 \cdot 5(3 \cdot 2)[n=24] \\
14 \cdot 1(3 \cdot 6)[n=26 \\
7 \cdot 7(1 \cdot 6)[n=27]\end{array}$ \\
\hline
\end{tabular}

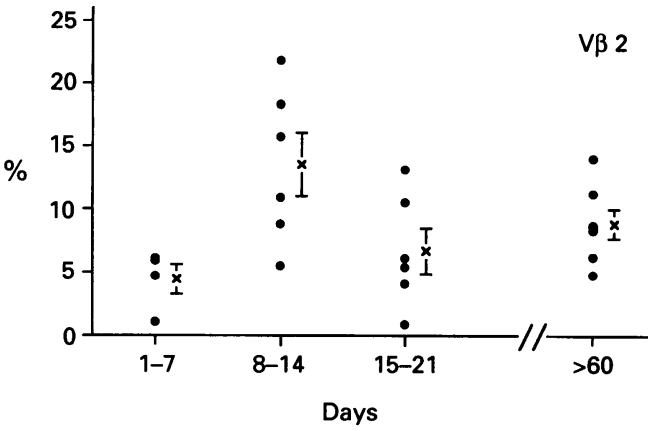

Figure $5 \quad V \beta 2$ expressing $T$ cells in patients with Kawasaki disease at different stages of the illness. The mean percentage of $V \beta 2$ expressing $T$ cells from Kawasaki disease patients in the second week of the illness was higher than that found in either the first $(p<0.05)$ or third $(p=0.05)$ week of the illness, or from Kawasaki disease patients in late convalescence $(p=0 \cdot 10)$. Each point represents an individual patient. Blood was not available from all patients at each time point and this graph comprises data from 18 patients. Data are included only where the exact date of onset of disease is known. Crosses represent means with SEM indicated by bars.

significantly increased above that in healthy controls $(25.5(4.6) v 10.0(1.4) \%, \mathrm{p}<0.005)$, but there was no significant difference between those with Kawasaki disease and children with other illnesses $(22 \cdot 5(3 \cdot 2) \%$, $\mathrm{p}=\mathbf{0 . 6 7 )}$ or the convalescent Kawasaki disease patients $(29 \cdot 2(7 \cdot 8) \%, p=0 \cdot 73)$ (fig 4).

Although the proportion of $\mathrm{V} \beta 5,12$, and 19 expressing $T$ cells in the patients with Kawasaki disease were similarly increased above that in healthy controls, there were no significant differences between children with Kawasaki disease and those with other illnesses or the convalescent patients with the disease (table).

When the percentage of V $\beta 2$ positive cells was assessed in relation to the timing of the sample after onset of the disease, the maximum rise in V $\beta 2$ expression was found to occur in the second week of the illness (fig 5).

\section{Discussion}

These results show that patients with Kawasaki disease have a selective increase in the proportion of PBMCs expressing V 32 . The expected rise in the proportion of $\mathrm{V} \beta 2$ bearing $T$ cells was induced by in vitro stimulation of normal PBMCs with toxic shock syndrome toxin, confirming the use of this method for detection of changes in V $\mathrm{2} 2$ expression.

These findings are in accord with those reported by Abe et al, who also showed selective $V \beta 2$ expression in children with Kawasaki disease, using a different methodology. ${ }^{24}$ In their study V $\beta$ expression was assessed after in vitro cultivation of $T$ cells in the presence of anti-CD3 antibodies, and after stimulation with IL-2. Monoclonal antibodies were used to detect the expressed proteins, and the polymerase chain reaction to detect production of V $\beta$ segments at the RNA level. Our results using direct staining of uncultured cells might more accurately reflect the situation in vivo.

The proportion of $T$ cells expressing V $\beta 5,8$, 12 , and 19 was found to be increased above that in healthy controls. However, there were no significant differences for these receptors 
when the patients with Kawasaki disease were compared with children with other illnesses.

These findings are in contrast to those of Abe et al, who reported a selective increase in the proportion of $\mathrm{V} \beta 8$ bearing $\mathrm{T}$ cells, in addition to $V \beta 2$ bearing $T$ cells, in patients with Kawasaki disease in North America. However, in their study V $\beta 8$ expressed in these patients, as detected by monoclonal antibodies, was only compared with that in healthy controls. ${ }^{24} 27$ The increase in V $\beta 8$ in patients observed in their study may be the result of febrile controls not being included in the comparison.

The finding of selective V 32 expression in the study of Abe et al and our own supports the hypothesis that Kawasaki disease is caused by a superantigen, similar to that responsible for the staphylococcal and streptococcal toxin diseases. ${ }^{24} 27$ The recent report by Leung et al of the isolation of toxic shock syndrome toxinproducing staphylococci or streptococcal pyrogenic exotoxin-secreting streptococci from children with Kawasaki disease is additional support for this hypothesis. ${ }^{28}$

However, the data also raise important questions. If Kawasaki disease is mediated by toxins related to toxic shock syndrome toxin, which selectively utilises V $\beta 2$, why is increased $\mathrm{V} \beta 2$ expression only detected in some of the patients? Failure to detect increased V $\beta 2$ expression in all the patients may be related to the timing of the sample in relation to the onset of the disease. Our finding, that V $\beta 2$ is most consistently increased in patients studied in the second week of the illness, suggests that detection of this phenomenon is critically dependent on the timing of the investigation. A study by Hibberd et al showed changes in V $\beta$ expression after tetanus immunisation in vivo followed a similar time course with a peak at two to three weeks followed by a decline and subsequent late increase. ${ }^{29}$ However, other explanations may be either that a single toxin may amplify different $V \beta$ receptor bearing $T$ cells in genetically defined subgroups of patients, or alternatively that more than one toxin may be involved, which binds to and amplifies different $V \beta$ receptor bearing $T$ cells. This possibility is supported by our finding that sterile supernatants from nose and throat cultures from patients with Kawasaki disease contain several different staphylococcal toxins, ${ }^{30}$ and the finding of Leung et al, that streptococcal exotoxins, rather that toxic shock syndrome toxin, were detected in some patients.

In conclusion, our data add to the growing evidence supporting a role for superantigen toxins in the aetiology of Kawasaki disease. A clear understanding of this role will depend on more detailed characterisation of these toxins.

Dr Nigel Curtis is a MRC research fellow; Dr Richard Zheng was supported by the British Heart Foundation.
1 Kawasaki T. Acute febrile mucocutaneous syndrome with lymphoid involvement with specific desquamation of the fingers and toes in children [Japanese]. Fpn $\mathcal{F}$ Allergy 1967; 16: $178-222$.

2 Dhillon R, Newton L, Rudd PT, Hall SM. Management of Kawasaki disease in the British Isles. Arch Dis Child 1993; 69: 631-8.

3 Bell DM, Morens DM, Holman RC, Hurwitz ES, Hunter MK. Kawasaki syndrome in the United States 1976 to 1980. Am $\mathcal{F}$ Dis Child 1983; 137: 211-4.

4 Yanagawa $\mathrm{H}$, Nakamura $\mathrm{Y}$, Yashiro $\mathrm{M}$, et al. A nationwide incidence survey of Kawasaki disease in 1985-1986 in Japan. $\mathcal{F}$ Infect Dis 1988; 158: 1296-301.

5 Kato $\mathrm{H}$, Koike S, Yamamoto M, Ito Y, Yano E. Coronary aneurysms in infants and young children with acute febrile mucocutaneous lymph node syndrome. $f$ Pediatr 1975 ; 86: 892-8.

6 Suzuki A, Tizard EJ, Gooch V, Dillon MJ, Haworth SG. Kawasaki disease: echocardiographic features in 91 cases presenting in the United Kingdom. Arch Dis Child 1990; 65: $1142-6$.

7 Nakamura Y, Fujita Y, Nagai $M$, et al. Cardiac sequelae of Kawasaki disease in Japan: statistical analysis. Pediatrics Kawasaki disease in

8 Tizard EJ, Suzuki A, Levin M, Dillon MJ. Clinical aspects of 100 patients with Kawasaki disease. Arch Dis Child 1991; 66: 185-8.

9 Nakamura Y, Yanagawa I, Kawasaki T. Temporal and geographical clustering of Kawasaki disease in Japan. Prog Clin Biol Res 1987; 250 (19): 19-32.

10 Yanagawa H, Nakamura Y, Kawasaki T, Shigematsu I. Nationwide epidemic of Kawasaki disease in Japan during winter of 1985-86. Lancet 1986; ii: 1138-9.

11 Fujita Y, Nakamura Y, Sakata K, et al. Kawasaki disease in families. Pediatrics 1989; 84: 666-9.

12 Furusho K, Ohba T, Soeda T, Kimoto K, Okabe T, Hirota $T$. Possible role for mite antigen in Kawasaki disease [letter]. Lancet 1981; ii: 194-5.

13 Hamashima Y, Kishi K, Tasaka K. Rickettsia-like bodies in infantile acute febrile mucocutaneous lymph-node syndrome Lancet 1973; ii: 42.

14 Kato $\mathrm{H}$, Fujimoto $\mathrm{T}$, Inoue $\mathrm{O}$, et al. Variant strain of Propionibacterium acnes: a clue to the aetiology of Kawasaki disease. Lancet 1983; ii: 1383-8.

15 Nigro $G$, Zerbini $M$, Krzysztofiak $A$, et al. Active or recent parvovirus B19 infection in children with Kawasaki disease. Lancet 1994; 343: 1260-1.

16 Shulman ST, Rowley AH, Fresco R, Morrison DC. The etiology of Kawasaki disease: retrovirus? Prog Clin Biol Res 1987; 250 (117): 117-2.

17 Burns JC, Geha RS, Schneeberger EE, et al. Polymerase activity in lymphocyte culture supernatants from patients with Kawasaki disease. Nature 1986; 323: 814-6.

18 Todd J, Fishaut $M$. Toxic shock syndrome associated with phage group 1 staphylococci. Lancet 1978; ii: 1116-8.

19 Torres-Martinez C, Mehta D, Butt A, Levin M Streptococcus associated toxic shock. Arch Dis Child 1992; 67: 126-30.

20 Cone LA, Woodward DR, Schlievert PM, Tomory GS. Clinical and bacteriological observations of a toxic shocklike syndrome due to Streptococcus pyogenes. $N$ Engl f Med 1987; 317: 146-9.

21 Marrack P, Kappler J. The staphylococcal enterotoxins and their relatives. Science 1990; 248: 705-11.

22 Choi YW, Herman A, DiGiusto D, Wade T, Marrack P, Kappler J. Residues of the variable region of the T-cellreceptor beta-chain that interact with $S$ aureus toxin superantigens. Nature 1990; 346: 471-3.

23 Levin M, Tizard EJ, Dillon MJ. Kawasaki disease: recent advances. Arch Dis Child 1991; 66: 1369-72.

24 Abe J, Kotzin BL, Jujo K, et al. Selective expansion of $\mathrm{T}$ cells expressing $\mathrm{T}$-cell receptor variable regions $\mathrm{V}$ beta 2 and V beta 8 in Kawasaki disease. Proc Natl Acad Sci USA 1992; 89: 4066-70.

25 Shackelford PG, Strauss AW. Kawasaki syndrome [editorial; comment]. N Engl $\mathcal{\text { Med } 1 9 9 1 ; 3 2 4 : 1 6 6 4 - 6 .}$ 26 Diagnostic guidelines for Kawasaki disease. American Heart Association Committee on rheumatic fever, endocarditis, and Kawasaki disease. Am $\mathcal{F}$ Dis Child 1990; 144: 1218-9.

27 Abe J, Kotzin BL, Meissner C, et al. Characterization of $\mathrm{T}$ cell repertoire changes in acute Kawasaki disease. f Exp Med 1993; 177: 791-6.

28 Leung DYM, Meissner HC, Fulton DR, Murray DL, Kotzin BL, Schlievert PM. Toxic shock syndrome toxinsecreting Staphylococcus aureus in Kawasaki syndrome. Lancet 1993; 342: 1385-8.

29 Hibberd ML, Wong FS, Nicholson LB, Demaine AG. Analysis of human $\mathrm{T}$-cell receptor $\mathrm{V}$-beta gene usage following immunization to tetanus toxoid in vivo. Immunology 1993; 79: 398-402.

30 Curtis N, Chan B, Levin M. Toxic shock syndrome toxinsecreting Staphylococcus aureus in Kawasaki syndrome [letter]. Lancet 1994; 343: 299. 